\title{
Ginkgo fruit extract as an additive to modify rumen microbiota and fermentation and to mitigate methane production
}

\author{
S. Oh, R. Shintani, S. Koike, and Y. Kobayashi ${ }^{1}$ \\ Graduate School of Agriculture, Hokkaido University, Sapporo 060-8589, Japan
}

\begin{abstract}
Ginkgo fruit, an unused byproduct of the ginkgo nut industry, contains antimicrobial compounds known as anacardic acids. Two major cultivars of ginkgo, Kyuju $(\mathrm{K})$ and Tokuro $(\mathrm{T})$, were evaluated for their potential as a feed additive for ruminants. In batch culture, we incubated a mixture of hay and concentrate in diluted rumen fluid with or without $1.6 \%$ (fruit equivalent) ginkgo fruit extract. We conducted another series of batch culture studies to determine the dose response of fermentation. We also conducted continuous culture using the rumen simulation technique (RUSITEC) with cultivar $\mathrm{K}$ and carried out a pure culture study to monitor the sensitivity of 17 representative rumen bacterial species to ginkgo extract and component phenolics. Although both K and T extracts led to decreased methane and increased propionate production, changes were more apparent with $\mathrm{K}$ extract, and were dose-dependent. Total gas production was depressed at doses $\geq 3.2 \%$, suggesting that $1.6 \%$ was the optimal supplementation level. In RUSITEC fermentation supplemented with $1.6 \%$ ginkgo K, methane decreased by $53 \%$ without affecting total gas or total VFA production, but with decreased acetate and increased propionate. Disappearance of dry matter, neutral detergent fiber, and acid detergent fiber were not affected by ginkgo, but ammonia levels were decreased. Quantitative PCR indicated that the abundance of protozoa, fungi, methanogens, and bacteria related to hydrogen and formate production decreased, but the abundance of bacteria related to propionate production increased. MiSeq analysis (Illumina Inc., San Diego, CA) confirmed these bacterial changes and identified archaeal community changes, including a decrease in Methanobrevibacter and Methanomassiliicoccaceae and an increase in Methanoplanus. Pure culture study results supported the findings for the above bacterial community changes. These results demonstrate that
\end{abstract}

Received August 26, 2016.

Accepted November 18, 2016.

${ }^{1}$ Corresponding author: kyas@anim.agr.hokudai.ac.jp ginkgo fruit can modulate rumen fermentation toward methane mitigation and propionate enhancement via microbial selection.

Key words: ginkgo fruit extract, methane reduction, rumen fermentation, rumen microbiota

\section{INTRODUCTION}

Ginkgo (Ginkgo biloba) is a so-called living fossil classified as a deciduous tree. Ginkgo is widely distributed in Asian countries, including China, Japan, and Korea. In these areas, ginkgo nuts are consumed as food and as medicine. In Japan, about 1,000 t/yr of ginkgo nuts are produced from 6 cultivars, which differ by harvest period and the shape and size of the nut. Ginkgo fruit is a byproduct of ginkgo nut production, but is not used due to its peculiar smell and the presence of nutritionally negative compounds, such as a vitamin $\mathrm{B}_{6}$ antagonist. Recently, however, interest has been growing in the functional compounds (such as flavonoids, terpene trilactones, and alkylphenols) present in ginkgo leaves (van Beek and Montoro, 2009) and ginkgo fruit (Yang et al., 2004). Of these biochemical groups, the alkylphenols, represented by anacardic acid, cardanol, and cardol, have been found to possess useful characteristics such as antitumor (Itokawa et al., 1987), antifeedant (Matsumoto and Sei, 1987), antistress (Rai et al., 2003), and antibacterial (Kubo et al., 1993) activities. More recently, studies using ginkgo fruit and leaves have assessed their use as a feed additive for broiler chickens (Zhang et al., 2012) and piglets (Zhou et al., 2015). Cashew nut shell liquid (CNSL), which contains similar alkylphenols, has made favorable alterations to rumen fermentation, namely a reduction in methane and an increase in propionate production through rumen microbial selection (Watanabe et al., 2010; Shinkai et al., 2012). Anacardic acids in CNSL consist of 3 molecules with $\mathrm{C} 15$ alkyl side chains differing in saturation (C15:1, C15:2, and C15:3), but anacardic acids in ginkgo consist of 3 molecules with different side-chain lengths (C13:0, C15:1, and C17:1). A previous report suggested that the length of the alkyl side chain of anacardic acid differentially affects antibacterial activ- 
ity toward methicillin-resistant Staphylococcus aureus (Muroi et al., 2004). A variety of anacardic acids occur in a few agricultural plants that await evaluation for a wide range of applications. Of such future applications, the use of ginkgo fruit in ruminant feed is one possibility. We hypothesized that ginkgo fruit containing anacardic acid can modulate rumen fermentation without negatively affecting feed digestion. Therefore, we investigated rumen parameters, including gas, VFA, ammonia, and microbes, to evaluate the potential of gingko fruit extract as a rumen modifier.

\section{MATERIALS AND METHODS}

\section{Rumen Fluid and Donor Cows}

Rumen content was collected from 2 ruminally fistulated Holstein cows fed twice a day $(0800$ and $1700 \mathrm{~h})$ with a $50 \%$ concentrate (Monster 18; Mercian, Tokyo, Japan), $50 \%$ orchardgrass hay diet at the experimental farm of Hokkaido University, Sapporo, Japan. The diet contained $18.2 \% \mathrm{CP}, 41.2 \% \mathrm{NDF}$, and 2.16 Mcal of $\mathrm{ME} / \mathrm{kg}$ on a DM basis. An equal amount of rumen content from both cows was mixed and placed in a bottle flushed with $\mathrm{N}_{2}$ gas, then transferred to the laboratory within $30 \mathrm{~min}$. The rumen content was strained through 2 layers of surgical gauze and used for in vitro experiments.

\section{Ginkgo Fruit and Extract}

We chose 2 major cultivars of ginkgo, Kyuju (K) and Tokuro $(\mathrm{T})$, as candidate materials based on availability from annual production. We obtained fresh fruit after separation of the nut at a ginkgo farm in Sobue town, Aichi Prefecture, Japan, a major ginkgo-nut-producing area. Ginkgo fruit (mashed) was immediately frozen at $-30^{\circ} \mathrm{C}$ and shipped to the laboratory. For extraction, $364 \mathrm{~g}$ of ginkgo fruit was suspended in $680 \mathrm{~mL}$ of $99.5 \%$ ethanol for $48 \mathrm{~h}$, centrifuged to obtain the supernatant, and then concentrated by a centrifugal evaporator for experimental use. This stock extract was diluted with $99.5 \%$ ethanol to set each experimental dosage of ginkgo extract, expressed as percent (wt/vol) of ginkgo fruit (wet weight equivalent) in the final culture.

\section{Batch Culture}

In vitro batch culture tests were performed as follows. Artificial saliva (McDougall, 1948) and strained rumen fluid were mixed in a 1:1 ratio (vol/vol), and $10 \mathrm{~mL}$ of the mixture was transferred to test tubes (180 mm length, $10 \mathrm{~mm}$ diameter) with a substrate that was a mixture of $0.14 \mathrm{~g}$ concentrate and $0.06 \mathrm{~g}$ orchardgrass hay, identical to the mixture given to the rumen content donor cows. Ginkgo extract or an equal volume of ethanol $(100 \mu \mathrm{L})$ was added to tubes, and the headspace was flushed with $\mathrm{N}_{2}$ gas, sealed with a butyl rubber stopper and plastic cap, and incubated at $39^{\circ} \mathrm{C}$ for $24 \mathrm{~h}$. For cultivar comparison, the dose of ginkgo was set at $1.6 \%$ in fruit equivalent. For doseresponse assays, supplementation levels of $0,0.8,1.6$, 3.2 , and $6.4 \%$ were tested. Incubation was in quintuplicate for cultivar comparison $(\mathrm{n}=5)$ and quadruplicate for dose-response assays $(\mathrm{n}=4)$. After incubation, total gas production was measured through a needleattached pressure gauge (Aф60B, GL Sciences, Tokyo, Japan), and gas samples were analyzed for $\mathrm{CO}_{2}, \mathrm{CH}_{4}$, and $\mathrm{H}_{2}$ using gas chromatography (Watanabe et al., 2010). Cultures were centrifuged, and the supernatant was used for VFA analysis. These batch culture experiments were carried out to select the ginkgo cultivar (K vs. T) with the more potent effect and then to evaluate the dose response of rumen fermentation parameters to the selected cultivar (ginkgo K; see results).

\section{Continuous Culture}

The rumen simulation technique (RUSITEC; Czerkawski and Breckenridge, 1977) was used to evaluate longer-term rumen responses to ginkgo extract from the selected cultivar. The fermentation system was equipped with 8 fermentors that each had a $650-\mathrm{mL}$ working capacity. The procedure for operation, including feeding and sampling, was as described by Watanabe et al. (2010). The rumen inoculum was a mixture of strained rumen fluid from 2 cows (as used for the batch culture studies) and artificial saliva at a 5:2 ratio. Incubation lasted $7 \mathrm{~d}$, consisting of $5 \mathrm{~d}$ for adaptation and $2 \mathrm{~d}$ for sampling. Artificial saliva ( $\mathrm{pH}$ 6.8) was continuously supplied using a peristaltic pump with a dilution rate of $0.5 \mathrm{vol} / \mathrm{d}$. The experimental diet, a ground mixture consisting of $9.1 \mathrm{~g}$ of concentrate and $3.9 \mathrm{~g}$ of orchardgrass hay, the same mixture used in the batch culture study, was fed by nylon bag to each fermentor every $24 \mathrm{~h}$. Eight fermentors were divided into 2 groups (4 fermentors per group), to which ginkgo $\mathrm{K}$ extract in ethanol or an equal volume of ethanol $(4.86 \mathrm{~mL})$ was supplemented at feeding time. The dose level of ginkgo was $1.6 \%$ in fruit equivalent. Each nylon bag was taken from the fermentor after $48 \mathrm{~h}$ of incubation and rinsed with $20 \mathrm{~mL}$ of artificial saliva to recover microbes adhering to feed particles. The artificial saliva used for rinsing was returned to the fermentor at each feeding time. Rumen fluid samples were taken at $3 \mathrm{~h}$ intervals from each fermentor directly through a pipette. Feeding, rinsing bags, and pipette-aided sampling were done under $\mathrm{CO}_{2}$ flushing to maintain an anaerobic atmosphere. 
Ruminal pH, VFA, and ammonia were analyzed using all individual samples taken at $3 \mathrm{~h}$ intervals. Disappearance of DM, NDF, and ADF were determined by analyzing feed residues in nylon bag samples taken before feeding for the last $2 \mathrm{~d}$. The microbial population was analyzed using culture samples before feeding for the last $2 \mathrm{~d}$. Fermentation gas was collected continuously in a plastic bag (Tedlar PDF; DuPont, Wilmington, DE) connected to each fermentor for the last $2 \mathrm{~d}$. Total gas produced was measured by spirometer (Sanshin Kogyo, Tokyo, Japan), and gas composition was analyzed by gas chromatography (Shimadzu, Kyoto, Japan).

\section{Pure Cultures}

We cultivated representative rumen bacteria to determine the MIC of ginkgo K extract and of the purified phenolic compounds found in ginkgo: 3 anacardic acids (C13:0, C15:1, and C17:1), cardanol (C15:1), and cardol (C15:1). The rumen bacteria tested were Ruminococcus flavefaciens C94 (ATCC 19208) and Ruminococcus albus 7 (ATCC 27210) as hydrogen- and formate-producing bacteria; Eubacterium ruminantium GA195 (ATCC 17233), Butyrivibrio fibrisolvens D1 (ATCC 19171), and Butyrivibrio proteroclasticus (ATCC 51982) as butyrate-producing bacteria; Lactobacillus ruminis RF1 (ATCC 27780) and Streptococcus bovis (ATCC 33317) as lactate-producing bacteria; Fibrobacter succinogenes S85 (ATCC 19169), Prevotella ruminicola 23 (ATCC 19189), Succinivibrio dextrinosolvens 24 (ATCC 19716), Ruminobacter amylophilus $\mathrm{H} 18$ (ATCC 27944), Succinimonas amyloytica $\mathrm{B}_{2} 4$ (ATCC 19206), Selenomonas ruminantium GA192 (ATCC 12561); and Megasphaera elsdenii LC1 (ATCC 25940) as succinate- or propionate-producing bacteria or both; and Clostridium sticklandii (ATCC 12662), Clostridium aminophilum (ATCC 49906), and Peptostreptococcus anaerobius (ATCC 27337) as hyper-ammonia-producing bacteria. Tested concentrations of ginkgo K extract were $0,0.05,0.1,0.2,0.4$, and $1.6 \%$, and of purified phenolic compounds were $0,1.56,3.13,6.25$, and $12.5 \mu \mathrm{g} /$ $\mathrm{mL}$. The extract at $1.6 \%$ was equivalent to $1.19,6.76$, and $2.67 \mu \mathrm{g} / \mathrm{mL}$ for anacardic acids C13:0, C15:1, and $\mathrm{C} 17: 1,0.27 \mu \mathrm{g} / \mathrm{mL}$ for caldanol C15:1, and $1.59 \mu \mathrm{g} / \mathrm{mL}$ for cardol C15:1, respectively. All these pure phenolics were obtained from Idemitsu Co. Ltd. (Tokyo, Japan) and Sigma-Aldrich (Saint Louis, MO). Growth of each bacterium was monitored spectrophotometrically at $660 \mathrm{~nm}$. To culture all bacteria except L. ruminis, we used medium containing rumen fluid. The medium was prepared with $50 \mathrm{~mL}$ of distilled water, $7.5 \mathrm{~mL}$ each of mineral solutions I and II (Bryant and Burkey, 1953), $30 \mathrm{~mL}$ of rumen fluid, $0.2 \mathrm{~g}$ of bactopeptone, $0.12 \mathrm{~g}$ of yeast extract, $0.1 \mathrm{~mL}$ of $0.1 \%$ resazurin, $0.1 \mathrm{~g}$ of L-cysteine $\mathrm{HCl}, 5 \mathrm{~mL}$ of $8 \% \mathrm{Na}_{2} \mathrm{CO}_{3}$, and $0.25 \mathrm{~g}$ of each glucose and cellobiose. The growth medium for $L$. ruminis was MRS (Oxoid, Basingstoke, UK) containing L-cysteine $\mathrm{HCl}$.

\section{Chemical Analysis}

We analyzed the major components of the experimental feeds, ginkgo fruit, its extract, and nylon bag residue using the methods of the AOAC (1990) and Van Soest et al. (1991). We analyzed the total polyphenol and total flavonoid contents using the Folin-Ciocalteu method (Makkar et al., 1993) and the aluminum chloride method (Dewanto et al., 2002), respectively. Alkylphenolics in ginkgo fruit and ginkgo fruit extract (anacardic acids, cardanol, and cardol) were quantified by HPLC as described by Watanabe et al. (2010). Gases $\left(\mathrm{H}_{2}, \mathrm{CH}_{4}\right.$, and $\left.\mathrm{CO}_{2}\right)$ from microbial fermentation in batch cultures and RUSITEC were analyzed using a GC-8A gas chromatograph (Shimadzu) equipped with parallel columns of Porapak Q (Waters, Milford, MA) and Molecular Sieve 13X (Restek, Bellefonte, PA), and a thermal conductivity detector. We analyzed VFA as briefly described below. Culture fluid was mixed with $25 \%$ meta-phosphoric acid at a 5:1 ratio, incubated overnight at $4^{\circ} \mathrm{C}$, and centrifuged at $10,000 \times g$ at $4^{\circ} \mathrm{C}$. Then, the supernatant, mixed with crotonic acid as internal standard, was injected to a GC-14B gas chromatograph (Shimadzu) equipped with an ULBON HR-20M fused silica capillary column $(0.53 \mathrm{~mm}$ i.d. $\times$ $30 \mathrm{~m}$ length, $3.0 \mu \mathrm{m}$ film; Shinwa, Kyoto, Japan) and a flame-ionization detector. Ammonia nitrogen concentration was spectrophotometrically determined using the indophenol reaction (Weatherburn, 1967).

\section{Microbial Analysis}

We took samples for microbiological analysis from each fermentor fluid at the time of withdrawal of the nylon bag (after rinsing but before feeding with a new nylon bag), so that both planktonic and feed-associated microbes were included. These samples were immediately frozen and kept at $-80^{\circ} \mathrm{C}$. Extraction of DNA for microbial analysis was performed using the repeated bead beating plus column $(\mathrm{RBB}+\mathrm{C})$ method described by $\mathrm{Yu}$ and Morrison (2004). The DNA was used for real-time PCR to quantify abundance of rumen representatives including total bacteria, total archaea, $R$. flavefaciens, $R$. albus, B. fibrisolvens, Treponema bryantii, S. bovis, F. succinogenes, Prevotella bryantii, P. ruminicola, S. dextrinosolvens, R. amylophilus, Anaerovibrio lipolytica, S. ruminantium and M. elsdenii. 
All quantitative PCR (qPCR) details such as primers, standards, PCR conditions, and calculations were as described by Koike et al. (2007) and Ohene-Adjei et al. (2008). For protozoa and fungi, details were as referred to by Sylvester et al. (2004) and Denman and McSweeney (2006), respectively. In brief, standard plasmid containing the respective target gene sequence was obtained by PCR cloning using a target-specific primer set. The copy number of each standard plasmid was calculated using the molecular weight of nucleic acid and the length (base pair) of the cloned standard plasmid as described by Koike et al. (2007). A LightCycler system and a FirstStart DNA master SYBR I reaction kit (Roche, Penzberg, Germany) were used with 10-fold serial dilutions of standard plasmid for the respective target (16S rDNA or $18 \mathrm{~S}$ rDNA sequence specific to each target microbe). We calculated microbial quantity using amplification curves obtained from both standard and sample. The specificity of PCR amplification was confirmed using melting curve analysis of the PCR products by increasing the temperature from $70^{\circ} \mathrm{C}$ to $95^{\circ} \mathrm{C}$ at a rate of $0.1^{\circ} \mathrm{C} / \mathrm{s}$. Microbial abundance was shown by copy number of rDNA for total bacteria, protozoa, and fungi, or by relative proportion in total bacterial copy number for total archaea and specific bacteria.

To comprehensively analyze the microbial community, we used DNA samples from RUSITEC for MiSeq analysis (Illumina Inc., San Diego, CA). Sequencing was performed by Hokkaido System Science Co. Ltd. (Sapporo, Japan). The V3 to V4 regions were amplified using 2 primer sets: S-D-Bact-0341-b-S-17 (5'-CCTACGGGNGGCWGCAG-3') and S-D-Bact-0785-a-A-21 (5'-GACTACHVGGGTATCTAATCC-3') for bacterial rDNA (Herlemann et al., 2011), and arch349F $\left(5^{\prime}\right.$-GYGCASCAGKCGMGAAW- $\left.3^{\prime}\right)$ and $\operatorname{arch} 806 \mathrm{R}$ (5'-GGACTACVSGGGTATCTAAT-3') for archaeal rDNA (Takai and Horikoshi, 2000). Polymerase chain reaction was carried out in a $50-\mu \mathrm{L}$ total volume: $10 \mu \mathrm{L}$ of $5 \times$ PrimeSTAR Buffer, $4 \mu \mathrm{L}$ of dNTP mixture $(2.5$ $\mathrm{m} M$ each), $0.5 \mu \mathrm{L}$ of PrimeSTAR HS DNA polymerase (Takara Bio Inc., Kusatsu, Japan), $1 \mu \mathrm{L}$ of each primer $(10 \mathrm{pmol} / \mu \mathrm{L}), 32.5 \mu \mathrm{L}$ of distilled $\mathrm{H}_{2} \mathrm{O}$, and $1 \mu \mathrm{L}$ of template DNA $(10 \mathrm{ng} / \mu \mathrm{L})$. The following PCR conditions were used: 30 cycles for bacteria and 40 cycles for archaea, consisting of denaturation at $98^{\circ} \mathrm{C}(10 \mathrm{~s})$, annealing at $55^{\circ} \mathrm{C}(15 \mathrm{~s})$, and extension at $72^{\circ} \mathrm{C}(30$ s). Amplicon sequencing was carried out using MiSeq as described by Caporaso et al. (2012). Data quality control and analyses were performed using the QIIME ver. 1.8.0 pipeline (Caporaso et al., 2010). Operational taxonomic units were generated from sequences clustered at a $97 \%$ similarity threshold using the UCLUST algorithm (Edgar, 2010). Chimeric sequences were removed from the analysis using the ChimeraSlayer algorithm (http://microbiomeutil.sourceforge.net/\#A_ CS). Taxonomy was assigned using the Greengenes database (ver. 13.8; McDonald et al., 2012) at a $90 \%$ similarity threshold. Difference in biodiversity between control and ginkgo extract was compared by $\alpha$ diversity metrics: Chao1, Shannon index, phylogenetic diversity whole tree, observed number of operational taxonomic units, and Good's coverage.

\section{Statistical Analysis}

The data from batch culture studies were averaged (cultivar comparison, $\mathrm{n}=5$; dose response, $\mathrm{n}=4$ ) for ANOVA using the GLM procedure of SPSS version 16.0 J (IBM, Tokyo, Japan). Tukey's method was employed for multiple comparison. Orthogonal contrasts were used to test linear and quadratic effects of dose level of ginkgo K extract. In the RUSITEC experiment, the data (including $\mathrm{pH}, \mathrm{VFA}$, and ammonia) were subjected to ANOVA using the MIXED procedure of SPSS with repeated measures. The model included the effect of treatment, time after feeding, their interaction, random effects of fermentors within treatments, and residual error. Time after feeding was used as the repeated measure. Data on gas, NDF, ADF, and DM disappearance, $\mathrm{qPCR}$ and next-generation sequencing (MiSeq) from samples taken at $0 \mathrm{~h}$ (before feeding) for $2 \mathrm{~d}$ from each fermentor were averaged and compared (control vs. ginkgo) by Student's $t$-test. Statistical significance was considered at $P<0.05$.

\section{RESULTS}

\section{Chemical Composition of Ginkgo Fruit}

The chemical composition of 2 cultivars of ginkgo fruit is shown in Table 1. The chemical content of the cultivars was very different: $\mathrm{K}$ had a higher percentage of NDF and ether extract, and lower nonstructural carbohydrate than $\mathrm{T}$; $\mathrm{K}$ also had a higher content of total flavonoids than $T$. Both cultivars contained 3 types of anacardic acids, which were classified by length of the alkyl side chain and the number of double bonds (C13:0, C15:1, and C17:1). Ginkgo fruit also had cardanol $(\mathrm{C} 15: 1)$ and cardol $(\mathrm{C} 15: 1)$. The total content of these alkylphenols was more than 5 times higher in $\mathrm{K}$ than in T. The chemical composition of the ethanol extract of ginkgo fruit was essentially similar to that of the fruit: that is, $\mathrm{K}$ extract showed higher contents of ether extract, total polyphenol, total flavonoids, and alkylphenols represented by anacardic acid than T ex- 
Table 1. Chemical composition of 2 different cultivars of ginkgo (Kyuju and Tokuro) and extract ${ }^{1}$

\begin{tabular}{lccrcc}
\hline & \multicolumn{2}{c}{ Ginkgo fruit } & & \multicolumn{2}{c}{ Ginkgo fruit extract $^{2}$} \\
\cline { 2 - 3 } \cline { 5 - 6 } Content & Kyuju & Tokuro & & Kyuju & Tokuro \\
\hline DM (g/kg) & 200 & 284 & & 36 & 88 \\
Components (g/kg of DM) & 66 & 61 & & 2 & 2 \\
CP & 38 & 55 & & 3 & 10 \\
Crude ash & 200 & 62 & & & \\
NDF & 338 & 689 & & 571 & 874 \\
NSC & 358 & 132 & & 424 & 113 \\
Ether extract & 38 & 35 & & 119 & 45 \\
Total polyphenol & 33 & 8 & & 83 & 11 \\
Total flavonoid & 0.272 & 0.050 & & 30.8 & 8.0 \\
Alkyl phenols & 0.026 & 0.005 & & 2.9 & 0.8 \\
Anacardic acid (C13:0) & 0.147 & 0.027 & & 16.7 & 4.4 \\
Anacardic acid (C15:1) & 0.058 & 0.011 & & 6.6 & 1.7 \\
Anacardic acid (C17:1) & 0.006 & 0.001 & & 0.7 & 0.1 \\
Cardanol (C15:1) & 0.035 & 0.005 & & 3.9 & 8.0 \\
Cardol (C15:1) & & & & & \\
\hline
\end{tabular}

${ }^{1}$ All values shown are the average of triplicate analysis.

${ }^{2}$ Extract was made using $0.67 \mathrm{~g}$ of fresh fruit $/ \mathrm{mL}$ of ethanol.

tract. Nonstructural carbohydrate content was higher in $\mathrm{T}$ extract than in $\mathrm{K}$ extract.

\section{Batch Culture Study}

A comparison of the potency of the 2 ginkgo cultivars for modulating rumen fermentation is shown in Table 2. Although extracts from both cultivars greatly decreased methane production, $\mathrm{K}$ showed greater potential than $\mathrm{T}$ (86 vs. 66\%). We observed hydrogen accumulation following the addition of both ginkgo extracts, but this finding was not significant. Total VFA concentration was not changed by either ginkgo extract compared with the control. The concentration and molar proportion of acetate was decreased by $\mathrm{K}$ but not by $\mathrm{T}$. Both ginkgo extracts enhanced propionate production. We observed lower butyrate production for K. Based on these observations, we selected $\mathrm{K}$ as the more potent additive for modulating rumen fermentation and used it for further study. The dose response of fermentation to ginkgo $\mathrm{K}$ extract is shown in Table 3 . Total gas production decreased at $\geq 3.2 \%$ supplementation. Methane production was linearly inhibited in a dose-dependent manner. Supplementation at $>3.2 \%$ was quite effective for decreasing methane, but hydrogen accumulation occurred at $\geq 3.2 \%$. Total VFA concentration was not affected by the ginkgo extract. Acetate production linearly decreased, but propionate production linearly increased, depending on dose. The concentration and molar proportion of butyrate decreased at higher levels $(\geq 3.2 \%)$. All parameters except $\mathrm{CO}_{2}$ production showed a linear increase or decrease in dose response. Based on the observation of a decrease in total gas production and accumulation of hydrogen at $\geq 3.2 \%$,

Table 2. Comparison of 2 different cultivars of ginkgo (Kyuju and Tokuro) in alteration of rumen fermentation

\begin{tabular}{|c|c|c|c|c|c|}
\hline \multirow[b]{2}{*}{ Parameter } & \multirow[b]{2}{*}{ Control } & \multicolumn{2}{|c|}{ Extract $^{1}$} & \multirow[b]{2}{*}{ SEM } & \multirow[b]{2}{*}{$P$-value } \\
\hline & & Kyuju & Tokuro & & \\
\hline Total gas (mL) & $11.38^{\mathrm{a}}$ & $10.60^{\mathrm{b}}$ & $11.78^{\mathrm{a}}$ & 0.17 & 0.003 \\
\hline $\mathrm{CO}_{2}(\mathrm{~mL})$ & $9.56^{\mathrm{c}}$ & $10.25^{\mathrm{b}}$ & $10.98^{\mathrm{a}}$ & 0.18 & $<0.001$ \\
\hline $\mathrm{CH}_{4}(\mathrm{~mL})$ & $1.81^{\mathrm{a}}$ & $0.26^{\mathrm{b}}$ & $0.62^{\mathrm{b}}$ & 0.20 & $<0.001$ \\
\hline $\mathrm{H}_{2}(\mathrm{~mL})$ & 0.01 & 0.09 & 0.17 & 0.03 & 0.154 \\
\hline Total VFA $(\mathrm{m} M)$ & $87.5^{\mathrm{ab}}$ & $86.6^{\mathrm{b}}$ & $93.5^{\mathrm{a}}$ & 1.18 & 0.022 \\
\hline Acetate $(\mathrm{m} M)$ & $47.7^{\mathrm{a}}$ & $39.8^{\mathrm{b}}$ & $44.3^{\mathrm{a}}$ & 0.99 & 0.001 \\
\hline Propionate $(\mathrm{m} M)$ & $23.8^{\mathrm{b}}$ & $29.9^{\mathrm{a}}$ & $28.9^{\mathrm{a}}$ & 0.75 & $<0.001$ \\
\hline n-Butyrate $(\mathrm{m} M)$ & $12.3^{\mathrm{b}}$ & $11.0^{\mathrm{c}}$ & $13.9^{\mathrm{a}}$ & 0.36 & $<0.001$ \\
\hline Acetate (molar \%) & $55.4^{\mathrm{a}}$ & $46.0^{\mathrm{b}}$ & $47.4^{\mathrm{a}}$ & 0.99 & $<0.001$ \\
\hline Propionate (molar \%) & $27.2^{\mathrm{b}}$ & $34.5^{\mathrm{a}}$ & $30.9^{\mathrm{a}}$ & 0.81 & $<0.001$ \\
\hline n-Butyrate (molar \%) & $14.1^{\mathrm{b}}$ & $12.7^{\mathrm{c}}$ & $14.9^{\mathrm{a}}$ & 0.26 & $<0.001$ \\
\hline
\end{tabular}

${ }^{\mathrm{a}-\mathrm{c}}$ Means within a row with different superscripts differ $(P<0.05)$.

${ }^{1}$ Supplementation was set at $1.6 \%$ (wt/vol) of ginkgo fruit in final culture. 
Table 3. Dose response of ginkgo (Kyuju) extract supplementation on gas and short-chain fatty acid production

\begin{tabular}{|c|c|c|c|c|c|c|c|c|}
\hline \multirow[b]{2}{*}{ Parameter } & \multicolumn{5}{|c|}{ Level of supplementation } & \multirow[b]{2}{*}{ SEM } & \multicolumn{2}{|c|}{ Contrast $^{1}$} \\
\hline & Control & $0.8 \%$ & $1.6 \%$ & $3.2 \%$ & $6.4 \%$ & & Linear & Quadratic \\
\hline Total gas (mL) & $14.24^{\mathrm{a}}$ & $14.92^{\mathrm{a}}$ & $14.19^{\mathrm{a}}$ & $11.83^{\mathrm{b}}$ & $11.59^{\mathrm{b}}$ & 0.33 & $<0.001$ & 0.010 \\
\hline $\mathrm{CH}_{4}(\mathrm{~mL})$ & $3.57^{\mathrm{a}}$ & $3.14^{\mathrm{a}}$ & $2.43^{\mathrm{b}}$ & $0.87^{\mathrm{c}}$ & $0.28^{\mathrm{d}}$ & 0.29 & $<0.001$ & 0.020 \\
\hline $\mathrm{H}_{2}(\mathrm{~mL})$ & $0.00^{\mathrm{c}}$ & $0.00^{\mathrm{c}}$ & $0.00^{\mathrm{c}}$ & $0.05^{\mathrm{b}}$ & $0.09^{\mathrm{a}}$ & 0.01 & $<0.001$ & 0.002 \\
\hline Total VFA $(\mathrm{m} M)$ & 119.1 & 119.2 & 117.2 & 111.8 & 107.6 & 1.43 & 0.004 & 0.270 \\
\hline n-Butyrate $(\mathrm{m} M)$ & $13.2^{\mathrm{a}}$ & $12.7^{\mathrm{a}}$ & $12.7^{\mathrm{a}}$ & $9.8^{\mathrm{b}}$ & $8.7^{\mathrm{b}}$ & 0.42 & $<0.001$ & 0.034 \\
\hline Acetate (molar \%) & $65.3^{\mathrm{a}}$ & $62.0^{\mathrm{b}}$ & $56.8^{\mathrm{c}}$ & $49.8^{\mathrm{d}}$ & $47.8^{\mathrm{d}}$ & 1.53 & $<0.001$ & 0.691 \\
\hline Propionate (molar \%) & $19.9^{\mathrm{e}}$ & $23.6^{\mathrm{d}}$ & $28.1^{\mathrm{c}}$ & $36.1^{\mathrm{b}}$ & $38.5^{\mathrm{a}}$ & 1.61 & $<0.001$ & 0.624 \\
\hline n-Butyrate (molar \%) & $11.1^{\mathrm{a}}$ & $10.7^{\mathrm{a}}$ & $10.8^{\mathrm{a}}$ & $8.7^{\mathrm{b}}$ & $8.1^{\mathrm{c}}$ & 0.28 & $<0.001$ & $<0.001$ \\
\hline
\end{tabular}

${ }^{a-e}$ Means within a row with different superscripts differ $(P<0.05)$.

${ }^{1} P$-value for the linear and quadratic effects of ginkgo extract supplementation at $0,0.8,1.6,3.2$, and $6.4 \%$ in ginkgo fruit equivalent in final culture.

we selected the $1.6 \%$ dose of ginkgo fruit equivalent for further evaluation.

\section{Continuous Culture Study}

Rumen fermentation profiles and feed digestibility in RUSITEC are shown in Table 4. The dosage used was $1.6 \%$, which was confirmed as effective without adverse alteration of rumen fermentation in batch cultures. Total gas and $\mathrm{CO}_{2}$ production was not affected, but methane production decreased by $47 \%$ when ginkgo extract was added. We observed a small amount of hydrogen accumulation. Ginkgo extract did not influence total VFA concentration. The concentration and molar proportion of acetate were decreased by ginkgo extract, but the corresponding values of propionate were increased. We observed no change in butyrate production. Ruminal $\mathrm{pH}$ was slightly but significantly decreased. Ammonia concentration deceased with ginkgo extract. Disappearance of DM, NDF, and ADF were not affected by the addition of ginkgo extract.

The absolute abundance of total bacteria expressed as log copy number per milliliter of culture increased in the presence of ginkgo extract, but that of protozoa and fungi decreased. Other bacteria, including archaea, were compared by relative abundance of total bacteria (Figure 1). Most of the groups quantified showed considerable changes with ginkgo extract supplementation: total archaea, $R$. flavefaciens, $R$. albus, T. bryantii, the genus Treponema, and $P$. bryantii decreased; genus Prevotella, P. ruminicola, S. dextrinosolvens, R. amylophilus, A. lipolytica, S. ruminantium, and M. elsdenii

Table 4. Effect of ginkgo (Kyuju) extract supplementation on rumen fermentation profile in rumen simulation technique (RUSITEC) culture

\begin{tabular}{lccrr}
\hline Parameter & Control & $\begin{array}{c}\text { Ginkgo (Kyuju) } \\
\text { extract }\end{array}$ & SEM & $P$-value \\
\hline Total gas $(\mathrm{mL} / \mathrm{d})$ & $1,625.0$ & $1,580.0$ & 80.86 & 0.797 \\
$\mathrm{CO}_{2}(\mathrm{~mL} / \mathrm{d})$ & $1,387.0$ & $1,442.1$ & 73.87 & 0.729 \\
$\mathrm{CH}_{4}(\mathrm{~mL} / \mathrm{d})$ & 233.0 & 109.2 & 16.55 & $<0.001$ \\
$\mathrm{H}_{2}(\mathrm{~mL} / \mathrm{d})$ & 5.0 & 28.9 & 3.87 & 0.003 \\
$\mathrm{pH}$ & 6.67 & 6.62 & 0.01 & $<0.001$ \\
Total VFA $(\mathrm{m} M)$ & 64.9 & 67.5 & 0.89 & 0.143 \\
Acetate $(\mathrm{m} M)$ & 30.1 & 23.0 & 0.45 & $<0.001$ \\
Propionate $(\mathrm{m} M)$ & 11.3 & 17.3 & 0.34 & $<0.001$ \\
n-Butyrate $(\mathrm{m} M)$ & 15.8 & 15.9 & 0.24 & 0.625 \\
Acetate $(\mathrm{molar} \%)$ & 49.2 & 37.9 & 0.71 & $<0.001$ \\
Propionate $(\mathrm{molar} \%)$ & 18.5 & 28.5 & 0.56 & $<0.001$ \\
n-Butyrate (molar \%) & 25.8 & 26.3 & 0.39 & 0.295 \\
Ammonia (mg of N/100 $\mathrm{mL})$ & 17.5 & 8.1 & 0.85 & $<0.001$ \\
DM digestibility $(\%)$ & 59.6 & 60.9 & 0.47 & 0.184 \\
NDF digestibility $(\%)$ & 46.7 & 46.3 & 0.47 & 0.718 \\
ADF digestibility $(\%)$ & 27.9 & 28.5 & 0.45 & 0.546 \\
\hline
\end{tabular}

${ }^{1}$ Ginkgo extract was supplemented at $1.6 \%$ of ginkgo fruit in final culture. 

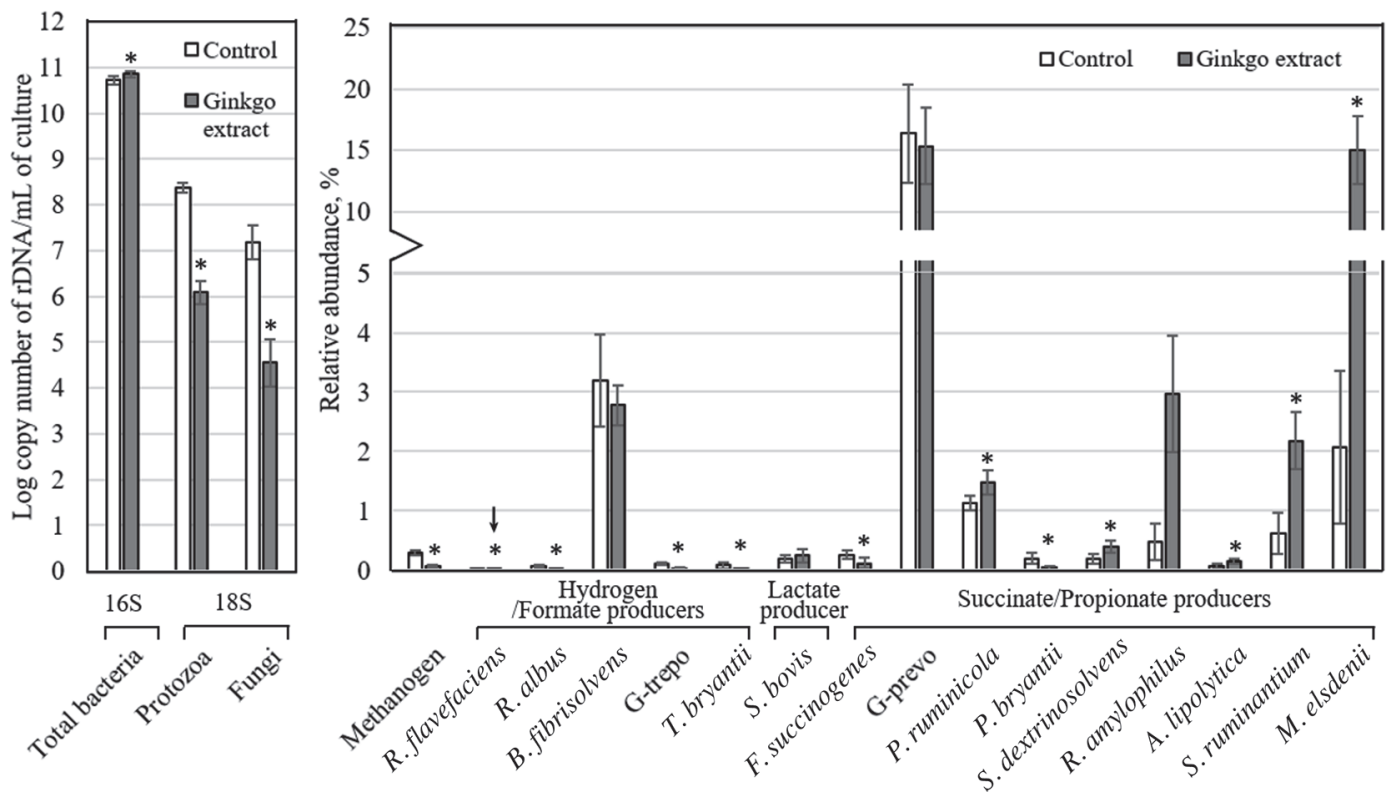

Figure 1. Relative abundance of rumen representative bacterial species and groups in RUSITEC (rumen simulation technique). Ginkgo extract was supplemented at $1.6 \%$ in fruit equivalent. G-trepo = genus Treponema, G-prevo = genus Prevotella; species include Ruminococcus flavefaciens, Ruminococcus albus, Butyrivibrio fibrisolvens, Treponema bryantii, Streptococcus bovis, Fibrobacter succinogenes, Prevotella ruminicola, Prevotella bryantii, Succinivibrio dextrinosolvens, Ruminobacter amylophilus, Anaerovibrio lipolytica, Selenomonas ruminantium, and Megasphaera elsdenii. Arrow indicates decrease. Asterisk $\left(^{*}\right)$ indicates statistical significance $(P<0.05)$. Error bars indicate SD.

increased. In particular, the abundance of M. elsdenii was about 7 times higher with ginkgo extract addition than with control.

Rumen microbiota were comprehensively analyzed by MiSeq and compared for control and treatment (ginkgo extract). The number of sequences passing the quality filter was 102,817 for control (minimum $=67,318 ;$ median $=85,305$ ) and 59,762 for treatment $($ minimum $=29,116 ;$ median $=66,186)$. For archaea, 57,253 sequences were obtained from samples of control $($ minimum $=31,844 ;$ median $=55,318)$ and 51,057 for treatment $($ minimum $=22,631 ;$ median $=53,841)$.
Changes in biodiversity indices inferred from bacterial and archaeal sequence reads are shown in Table 5. The high levels of Good's coverage suggest that sequences from the control and treatment represented the majority of bacteria and archaea present in the cultures. The richness and evenness of the bacterial and archaeal community decreased with treatment. The total number of operational taxonomic units, Chaol and Shannon indices, and phylogenetic diversity (PD_Whole_tree) for both bacteria and archaea were lowered by treatment. Thus, all indices for biodiversity decreased with ginkgo treatment.

Table 5. Comparison of bacterial and archaeal biodiversity by MiSeq analysis (Illumina Inc., San Diego, CA) of rumen simulation technique (RUSITEC) culture

\begin{tabular}{lcccr}
\hline Item & Control & $\begin{array}{c}\text { Ginkgo (Kyuju) } \\
\text { extract }\end{array}$ & SEM & $P$-value \\
\hline Bacterial community & & & & \\
Operational taxonomic units & 4,706 & 1,728 & 587 & 0.007 \\
Chao1 & 9,730 & 3,505 & 1,236 & 0.010 \\
Shannon (H') & 8.3 & 5.5 & 0.5 & $<0.001$ \\
PD_whole_tree & 261 & 118 & 28 & 0.003 \\
Good's coverage (\%) & 97.2 & 98.2 & 0.2 & 0.021 \\
Archaeal community & 1,398 & 761 & 141 & 0.024 \\
Operational taxonomic units & 2,910 & 1,423 & 310 & 0.013 \\
Chao1 & 4.5 & 2.8 & 0.3 & $<0.001$ \\
Shannon (H') & 51 & 34 & 4 & 0.019 \\
PD_whole_tree & 98.6 & 99.2 & 0.1 & 0.036 \\
Good's coverage (\%) & & & & \\
\hline
\end{tabular}

${ }^{1}$ Ginkgo extract was supplemented at $1.6 \%$ of ginkgo fruit in final culture. 
Changes in bacterial and archaeal communities at different taxonomic levels with ginkgo extract supplementation are shown in Figure 2. For bacteria at the phylum level (Figure 2A), the abundance of Bacteroidetes decreased with ginkgo extract (43.3 vs. $24.1 \%$ ), but the abundance of Firmicutes increased (35.3 vs. $44.0 \%$ ). The abundance of Proteobacteria was 4.6 times higher with ginkgo extract (3.9 vs. 18.0\%). More drastic change was seen for Synergistes, showing a 41 -fold increase with ginkgo extract (0.1 vs. $4.6 \%$ ), of which Pyramidobacter was the main member. At the genus level (Figure 2B), increased abundance with ginkgo extract was seen for specific groups related to succinate and propionate production, or both. These were Succinivibrio (0.6 vs. 9.8\%), Ruminobacter (2.2 vs. $7.2 \%$ ), Selenomonas (0.5 vs. $4.1 \%$ ), Megasphaera (6.7 vs. $22.6 \%$ ), and Prevotella (14.5 vs. $19.4 \%$ ). At the same time, decreased abundance was seen for Fibrobacter (2.5 vs. $0.7 \%$ ) and unclassified bacteria belonging to the Bacteroidales (19.7 vs. 1.6\%), Clostridiales (4.4 vs. $0.4 \%$ ), and Ruminococcaceae (6.6 vs. $0.4 \%$ ). The archaea community in the control comprised 3 classes: Methanobacteria (40.1\%), Methanomicrobia (25.6\%), and Thermoplasmata (19.7\%). By adding ginkgo extract, the community was changed toward more Methanomicrobia (61.4\%) and less Methanobacteria (17.2\%) and Thermoplasmata $(0.6 \%)$ (Figure 2C). The majority of each class were the genera Methanobrevibacter (39.3 vs. $16.9 \%$ ), Methanoplanus (22.3 vs. $59.8 \%$ ), and vadin CA11 (Methanomassiliicoccaceae; 19.7 vs. 0.6\%; Figure 2D).

\section{Pure Culture Study}

The MIC values of ginkgo extract and its phenolic composition for rumen bacteria are shown in Table 6 .
The bacterial response to ginkgo extract was clearly divided into sensitive (MIC $\leq 0.1 \%$ ) and insensitive (MIC $>1.6 \%$ ) groups. The sensitive group included $R$. flavefaciens, R. albus, E. ruminantium, B. fibrisolvens, B. proteoclasticus, S. bovis, F. succinogenes, P. ruminicola, S. amyloytica, C. sticklandii, C. aminophilum, and $P$. anaerobius. Insensitive species were $L$. ruminis, $S$. dextrinosolvens, $R$. amylophilus, S. ruminantium, and M. elsdenii. Hydrogen/formate producers and hyperammonia-producing bacteria belonged to the sensitive group, but succinate- and propionate-producing (or both) bacteria were in the insensitive group. Purified cardanol and cardol, phenolic compounds present in ginkgo, did not show strong antibacterial activity to rumen bacteria except $C$. sticklandii, but all 3 anacardic acids showed selective inhibition of bacterial growth, although the anacardic acid with a C17:1 alkyl side chain was slightly less potent than the others (C13:0 and $\mathrm{C} 15: 1$ ). Of the hyper-ammonia-producing bacteria, C. sticklandii and $P$. anaerobius were sensitive to ginkgo extract and the purified phenolic compounds, but $C$. aminophilum was less sensitive.

\section{DISCUSSION}

\section{Alkylphenols and Other Compounds of Ginkgo Fruit}

Of the alkylphenols, anacardic acid was most abundantly present in ginkgo fruit and its extract (Table 1). Although the alkyl side-chain structure is different, the anacardic acids in ginkgo were potent for inhibiting specific rumen bacteria (Table 6), as found for the anacardic acids in CNSL (Watanabe et al., 2010). This suggests that ginkgo fruit is as potent as CNSL in altering the rumen microbial community via the surfactant action of alkylphenols (Kubo et al., 1993) to
(A)

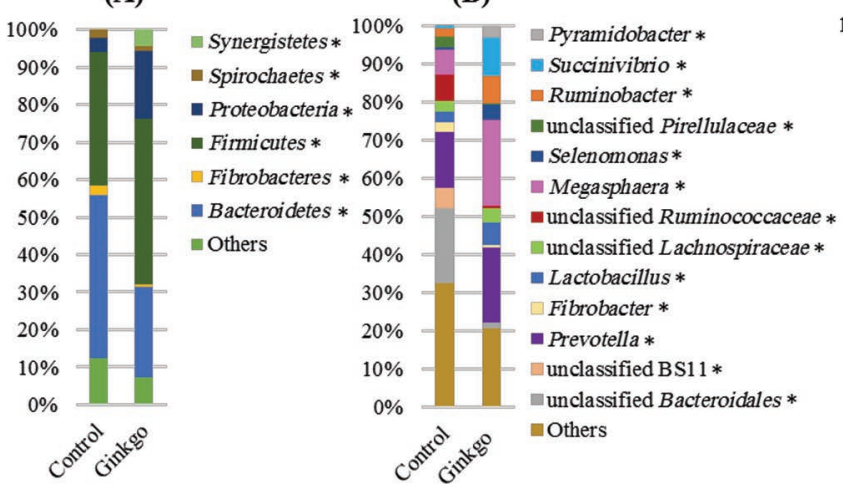

(C)

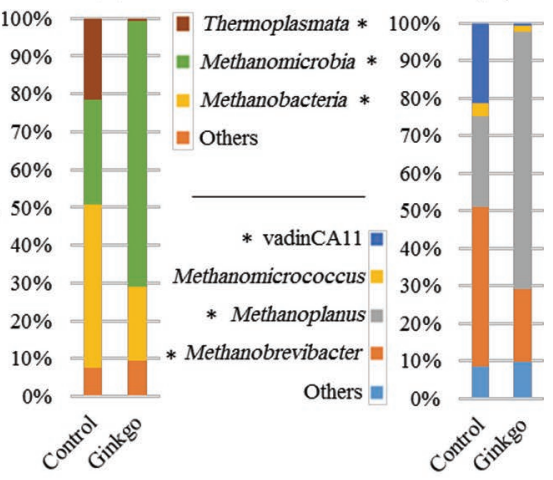

Figure 2. Effect of ginkgo extract supplementation on rumen microbiota in RUSITEC (rumen simulation technique) evaluated by MiSeq (Illumina Inc., San Diego, CA) at bacterial phylum level (A), at bacterial genus level (B), at archaeal class level (C), and at archaeal genus level (D). Asterisk $(*)$ indicates statistical significance between control and treatment $(P<0.05)$. 
lead fermentation changes, if dosed at a suitable level (Watanabe et al., 2010; Shinkai et al., 2012).

Ginkgo fruit contains a greater proportion of anacardic acids than other parts (leaves or seeds) that have been commercially used (van Beek and Montoro, 2009). Therefore, the fruit is more functional as an antimicrobial and more easily available in terms of quantity than leaves and seeds. As predicted, we observed a difference in functionality between ginkgo cultivars (Table 2 ), and we identified K, widely distributed as the main cultivar, as a better feed additive candidate for ruminant animals. Compared with ginkgo $\mathrm{K}$, ginkgo $\mathrm{T}$ had a lower alkylphenol content and a higher nonstructural carbohydrate content, including polysaccharides that might interact with alkylphenols, reducing the potency of such phenols, or more simply, making phenols physically less available for microbiota (Cieslak et al., 2014). This could be why $\mathrm{T}$ was less potent than K. Based on total flavonoid and nonstructural carbohydrate contents, other bioactive compounds besides alkylphenols in ginkgo extract might influence rumen fermentation; for example, flavonoid-rich plant extracts decrease rumen methane (Kim et al., 2015), and ginkgo leaf polysaccharides possess antioxidant activity (Jiao et al., 2016). More details on the mode of action of ginkgo extract is needed, but alkylphenols could be a main factor in altering rumen microbiota and fermentation (discussed later). In application, one problem needs to be solved: ginkgo fruit contains the nutritionally harmful 4-meth- ylpyridoxin (a vitamin $\mathrm{B}_{6}$ antagonist) (Isah, 2015), which may be unsuitable for animal feed. If the activity of this antinutritional compound can be reduced by biological treatments such as silage fermentation, then ginkgo fruit can be further evaluated in animal feed studies. Some lactic acid bacteria can produce vitamin $\mathrm{B}_{6}$ (Champagne et al., 2010), which might compensate for the negative effects of 4-methylpyridoxin if those bacteria are used as a silage inoculant. This possibility needs to be confirmed to better assess the functionality of ginkgo fruit as a potential feed additive for ruminant animals.

\section{Rumen Fermentation and Digestion}

Based on the RUSITEC study results, supplementation with ginkgo fruit extract altered the rumen fermentation pattern with no adverse effect on feed digestion (Table 4). We observed a significant decrease in methane production without affecting total gas production. Although the VFA pattern was greatly changed by ginkgo extract toward less acetate and more propionate production, total VFA levels were not changed. At the same time, and most importantly, the disappearance of DM, NDF, and ADF were not affected by ginkgo extract. This is the most addressed characteristic for developing a new additive candidate, as reported for CNSL (Watanabe et al., 2010). Indeed, such great shifts in rumen fermentation products are suggestive of

Table 6. Minimum inhibitory concentrations of ginkgo (Kyuju) extract for rumen bacteria

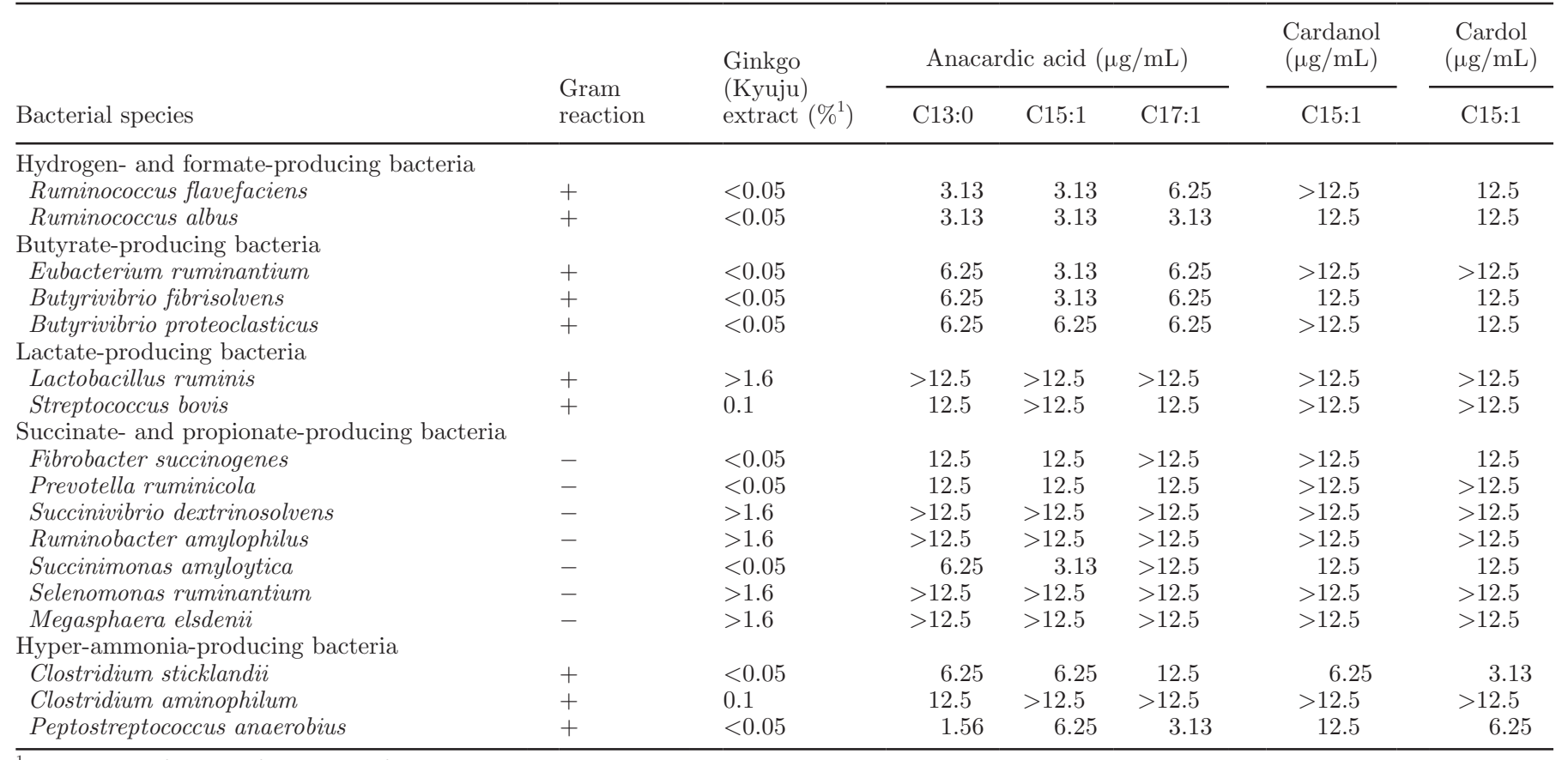

${ }^{1}$ Percentage of ginkgo fruit in the final culture. 
the occurrence of rumen microbial changes (see below). The selective inhibition of specific microbes by these additives should be compensated for by activation of alternative microbes to maintain rumen fermentation within normal conditions. Hydrogen accumulation with ginkgo supplementation was observed but was minimal, suggesting the quick development of an electron sink pathway alternative to methanogenesis, such as propionate production (Kobayashi, 2010). However, it is important to optimize the supplementation level of ginkgo extract, because an overdose $(>3.2 \%$ as fruit equivalent) can depress rumen fermentation, as shown in our dose-response experiments, where total gas production was inhibited (Table 3 ).

The rumen ammonia level was markedly reduced $(53.7 \%)$ by supplementation with ginkgo extract in RUSITEC (Table 4). In a similar RUSITEC study (Watanabe et al., 2010), CNSL decreased rumen ammonia level by $16.5 \%$. The decreased rumen ammonia level might lead to the improvement of feed $\mathrm{N}$ economy if the ammonia level is higher than the critical level for maintaining microbial protein synthesis $(5 \mathrm{mg}$ of N/dL; Griswold et al., 2003). The ammonia $\mathrm{N}$ level in the ginkgo-supplemented fermentor was $8.1 \mathrm{mg}$ of $\mathrm{N} /$ $\mathrm{dL}$, which could meet this requirement. The observed decreased ammonia level could be related to the abundance of hyper-ammonia-producing bacteria, including $P$. anaerobius, C. sticklandii, and C. aminophilum (Paster et al., 1993). In fact, they were quite sensitive to ginkgo extract and its component anacardic acids (Table 6). Protozoa contribute to ammonia production in the rumen (Patra and $\mathrm{Yu}, 2014$ ). Therefore, ammonia reduction observed in RUSITEC supplemented with ginkgo extract could be partly related to the decrease in protozoal population size (Figure 1).

Overall, although rumen responses to ginkgo extract were quite apparent, these observations were based on short-term incubation (up to $7 \mathrm{~d}$ in RUSITEC). Longer incubation or feeding studies are needed.

\section{Microbial Community}

Rumen fermentation changes due to ginkgo extract were reasonably attributable to microbial community changes evaluated by qPCR (Figure 1) and MiSeq (Figure 2) analyses, and by sensitivity of individual rumen bacteria to the extract (Table 6). Microbial selection by ginkgo extract was suggested by growth inhibition of hydrogen- and formate-producing bacteria ( $R$. flavefaciens and $R$. albus) and by growth stimulation of succinate- or propionate-producing bacteria $(P$. ruminicola, S. dextrinosolvens, R. amylophilus, S. ruminantium, and M. elsdenii) according to qPCR and MIC results. The MiSeq results confirmed this. Although butyrate producers (E. ruminantium, B. fibrisolvens, and $B$. proteoclasticus) were quite sensitive to ginkgo extract, as seen by the MIC, butyrate production in batch cultures or RUSITEC was not decreased. This was likely due to the increased abundance of $M$. elsdenii, which produce butyrate as another metabolite. The qPCR-observed decrease in number of protozoa and fungi, which are hydrogen and formate producers, respectively, is also indicative of these fermentation changes. Although the abundance of fibrolytic organisms (Fibrobacter, Ruminococcus, fungi) was depressed by ginkgo extract supplementation, NDF and ADF digestibility was not affected. This suggests development of an alternative fibrolytic system, possibly due to an increase in abundance of Prevotella and Selenomonas (Figure 2), some groups of which are involved in fiber degradation (Bekele et al., 2010; Sawanon et al., 2011). The contribution of uncharacterized fibrolytic bacteria (Kobayashi 2006; Jami et al., 2012) may also need to be considered. Although reduced protozoa and fungi may cause the decrease of fiber degradation, protozoa maintained in RUSITEC were non-fibrolytic entodinia (Béra-Maillet et al., 2005), and fungi might have already been selected for less fibrolytic ones in the $70 \%$ concentrate diet used in this study. Because the microbial situation influenced by ginkgo may differ between diets, evaluation of a high-fiber diet needs to be conducted to draw more general conclusions.

The decrease in methane with ginkgo extract supplementation in RUSITEC could be explained not only by a shift in the hydrogen-utilizing pathway to facilitate fumarate reduction and the acrylate pathway (both responsible for propionate production) but also by partial inhibition of methanogens. Decreased abundance of total methanogens, protozoa, and fungi by ginkgo (Figure 1) indicates both direct and indirect inhibition of methanogenesis, because planktonic methanogens and eukaryote (protozoa and fungi)-associated methanogens (Vogels et al., 1980; Cheng et al., 2009) are involved in methane synthesis. From MiSeq analysis for rumen archaea, 2 dominant genera were identified and showed different responses to ginkgo extract in their relative abundance: a decrease in abundance of Methanobrevibacter and an increase in Methanoplanus. Our data (unpublished results) suggest that sensitivity of methanogens to anacardic acids depends on the genus and even the species within the same genus. Because anacardic acids act as surfactants, differences in the cell surface structure of rumen methanogens might result in differential sensitivity. The Methanoplanus petrolearius type strain has glycoprotein in its cell envelope (Brambilla et al., 2010), which may confer tolerance to surfactants such as anacardic acids. This indicates the occurrence of archaeal selection. However, the contribu- 
tion of Methanoplanus to methane production is still unclear. Another interesting change due to supplementation with ginkgo extract was the decrease in number of Methanomassiliicoccaceae. Attention is being paid to this group of methanogens because of their contribution to methane production based on meta-transcriptomic analysis of cattle rumen (Poulsen et al., 2013); methylcoenzyme M reductase transcripts from this group decrease with dietary supplementation of rapeseed oil to decrease methane production. Ivy fruit saponin, another rumen modifier candidate, also reportedly decreases the abundance of this group of methanogens with a concomitant decrease of methane in RUSITEC (Belanche et al., 2016). The Methanomassiliicoccaceae may be a good target for mitigating rumen methane in the future strategy.

\section{CONCLUSIONS}

We evaluated the potential of ginkgo fruit as a feed additive in a series of in vitro experiments. Ginkgo extract obtained from the Kyuju cultivar significantly decreased ruminal methane and ammonia, and it enhanced propionate production. These effects were caused by selective inhibition of specific rumen microbes via surfactant action of the alkylphenols present in ginkgo fruit. Therefore, ginkgo fruit is a potential candidate as a feed additive for ruminant animals.

\section{ACKNOWLEDGMENTS}

This study was supported in part by Grant-in-Aid for Challenging Exploratory Research (number 26660207 to YK) from the Ministry of Education, Culture, Sports, Science and Technology, Japan. None of the authors has any financial or personal relationships that could inappropriately influence or bias the content of this report.

\section{REFERENCES}

AOAC. 1990. Official Methods of Analysis. 15th ed. Assoc. Off. Anal. Chem., Arlington, VA.

Bekele, A. Z., S. Koike, and Y. Kobayashi. 2010. Genetic diversity and diet specificity of ruminal Prevotella revealed by $16 \mathrm{~S}$ rRNA genebased analysis. FEMS Microbiol. Lett. 305:49-57.

Belanche, A., E. Pinloche, D. Preskett, and C. J. Newbold. 2016. Effects and mode of action of chitosan and ivy fruit saponins on the microbiome, fermentation and methanogenesis in the rumen simulation technique. FEMS Microbiol. Ecol. 92:1-13.

Béra-Maillet, C., E. Devillard, M. Cezette, J. P. Jouany, and E. Forano. 2005. Xylanases and carboxymethylcellulases of the rumen protozoa Polyplastron multivesiculatum, Eudiplodinium maggii and Entodinium sp. FEMS Microbiol. Lett. 244:149-156.

Brambilla, E., O. D. N. Djao, H. Daligault, A. Lapidus, S. Lucas, N. Hammon, M. Nolan, H. Tice, J. F. Cheng, C. Han, R. Tapia, L. Goodwin, S. Pitluck, K. Liolios, N. Ivanova, K. Mavromatis,
N. Mikhailova, A. Pati, A. Chen, K. Palaniappan, M. Land, L. Hauser, Y. J. Chang, C. D. Jeffries, M. Rohde, S. Spring, J. Sikorski, M. Göker, T. Woyke, J. Bristow, J. A. Eisen, V. Markowitz, P. Hugenholtz, N. C. Kyrpides, and H. P. Klenk. 2010. Complete genome sequence of Methanoplanus petrolearius type strain (SEBR $4847^{\mathrm{T}}$ ). Stand. Genomic Sci. 3:203-211.

Bryant, M. P., and L. A. Burkey. 1953. Cultural methods and some characteristics of some of the more numerous groups of bacteria in the bovine rumen. J. Dairy Sci. 36:205-217.

Caporaso, J. G., J. Kuczynski, J. Stombaugh, K. Bittinger, F. D. Bushman, E. K. Costello, N. Fierer, A. G. Peña, J. K. Goodrich, J. I. Gordon, G. A. Huttley, S. T. Kelley, D. Knights, J. E. Koenig, R. E. Ley, C. A. Lozupone, D. McDonald, B. D. Muegge, M. Pirrung, J. Reeder, J. R. Sevinsky, P. J. Turnbaugh, W. A. Walters, J. Widmann, T. Yatsunenko, J. Zaneveld, and R. Knight. 2010. QIIME allows analysis of high-throughput community sequencing data. Nat. Methods 7:335-336.

Caporaso, J. G., C. L. Lauber, W. A. Walters, D. Berg-Lyons, J. Huntley, N. Fierer, S. M. Owens, J. Betley, L. Fraser, M. Bauer, N. Gormley, J. A. Gilbert, G. Smith, and R. Knight. 2012. Ultrahigh-throughput microbial community analysis on the Illumina HiSeq and MiSeq platforms. ISME J. 6:1621-1624.

Champagne, C. P., T. A. Tompkins, N. D. Buckley, and J. M. GreenJohnson. 2010. Effect of fermentation by pure and mixed cultures of Streptococcus thermophilus and Lactobacillus helveticus on isoflavone and B-vitamin content of a fermented soy beverage. Food Microbiol. 27:968-972.

Cheng, Y. F., J. E. Edwards, G. G. Allison, W.-Y. Zhu, and M. K. Theodorou. 2009. Diversity and activity of enriched ruminal cultures of anaerobic fungi and methanogens grown together on lignocellulose in consecutive batch culture. Bioresour. Technol. 100:4821-4828.

Cieslak, A., P. Zmora, A. Stochmal, L. Pecio, W. Oleszek, E. PersKamczyc, J. Szczechowiak, A. Nowak, and M. Szumacher-Strabel. 2014. Rumen antimethanogenic effect of Saponaria officinalis L. phytochemicals in vitro. J. Agric. Sci. 152:981-993.

Czerkawski, J. W., and G. Breckenridge. 1977. Design and development of a long-term rumen simulation technique (Rusitec). Br. J. Nutr. 38:371-384.

Denman, S. E., and C. S. McSweeney. 2006. Development of a realtime PCR assay for monitoring anaerobic fungal and cellulolytic bacterial populations within the rumen. FEMS Microbiol. Ecol. $58: 572-582$.

Dewanto, V., X. Wu, K. K. Adom, and R. H. Liu. 2002. Thermal processing enhances the nutritional value of tomatoes by increasing total antioxidant activity. J. Agric. Food Chem. 50:3010-3014.

Edgar, R. C. 2010. Search and clustering orders of magnitude faster than BLAST. Bioinformatics 26:2460-2461.

Griswold, K. E., G. A. Apgar, J. Bouton, and J. L. Firkins. 2003. Effects of urea infusion and ruminal degradable protein concentration on microbial growth, digestibility, and fermentation in continuous culture. J. Anim. Sci. 81:329-336.

Herlemann, D. P. R., M. Labrenz, K. Jürgens, S. Bertilsson, J. J. Waniek, and A. F. Andersson. 2011. Transition in bacterial communities along the $2000 \mathrm{~km}$ salinity gradient of the Baltic Sea. ISME J. 5:1571-1579.

Isah, T. 2015. Rethinking Ginkgo biloba L.: Medicinal uses and conservation. Pharmacogn. Rev. 9:140-148.

Itokawa, H., N. Totsuka, K. Nakahara, K. Takeya, J. P. Lepoittevin, and Y. Asakawa. 1987. Antitumor principles from Ginkgo biloba L. Chem. Pharm. Bull. (Tokyo) 35:3016-3020.

Jami, E., A. Shabtay, M. Nikbachat, E. Yosef, J. Miron, and I. Mizarhi. 2012. Effects of adding a concentrated pomegranate-residue extract to the ration of lactating cows on in vivo digestibility and profile of rumen bacterial population. J. Dairy Sci. 95:5996-6005.

Jiao, R., Y. Liu, H. Gao, and K. F. So. 2016. The anti-oxidant and antitumor properties of plant polysaccharides. Am. J. Chin. Med. 44:463-488.

Kim, E. T., L. L. Guan, S. J. Lee, S. M. Lee, S. S. Lee, I. D. Lee, S. K. Lee, and S. S. Lee. 2015. Effects of flavonoid-rich plant extracts on in vitro ruminal methanogenesis, microbial populations 
and fermentation characteristics. Asian-australas. J. Anim. Sci. $28: 530-537$.

Kobayashi, Y. 2006. Inclusion of novel bacteria in rumen microbiology: Need for basic and applied science. Anim. Sci. J. 77:375-385.

Kobayashi, Y. 2010. Abatement of methane production from ruminants: Trends in the manipulation of rumen fermentation. Asianaustralas. J. Anim. Sci. 23:410-416.

Koike, S., H. Yabuki, and Y. Kobayashi. 2007. Validation and application of real-time polymerase chain reaction assays for representative rumen bacteria. Anim. Sci. J. 78:135-141.

Kubo, I., H. Muroi, and M. Himejima. 1993. Structural-antibiotic activity relationships of anacardic acids. J. Agric. Food Chem. 41:1016-1019.

Makkar, H. P. S., M. Blummel, N. K. Borowy, and K. Becker. 1993. Gravimetric determination of tannins and their correlations with chemical and protein precipitation method. J. Sci. Food Agric. 61:161-165.

Matsumoto, T., and T. Sei. 1987. Antifeedant activities of Ginkgo biloba L. components against the larva of Pieris rapae crucivora. Agric. Biol. Chem. 51:249-250.

McDonald, D., M. N. Price, J. Goodrich, E. P. Nawrocki, T. Z. DeSantis, A. Probst, G. L. Andersen, R. Knight, and P. Hugenholtz. 2012. An improved Greengenes taxonomy with explicit ranks for ecological and evolutionary analyses of bacteria and archaea. ISME J. 6:610-618.

McDougall, E. I. 1948. Studies on ruminant saliva. 1. The composition and output of sheep's saliva. Biochem. J. 43:99-109.

Muroi, H., K. Nihei, K. Tsujimoto, and I. Kubo. 2004. Synergistic effect of anacardic acids and methicillin against methicillin resistant Staphylococcus aureus. Bioorg. Med. Chem. 12:583-587.

Ohene-Adjei, S., A. V. Chaves, T. A. McAllister, C. Benchaar, R. M Teather, and R. J. Forster. 2008. Evidence of increased diversity of methanogenic archaea with plant extract supplementation. Microb. Ecol. 56:234-242.

Paster, B. J., J. B. Russell, C. M. J. Yang, J. M. Chow, C. R. Woese, and R. Tanner. 1993. Phylogeny of ammonia-producing ruminal bacteria, Peptostreptococcus anaerobius, Clostridium sticklandii and Clostridium aminophilum sp. nov. Int. J. Syst. Bacteriol. 43:107-110.

Patra, A. K., and Z. Yu. 2014. Effects of vanillin, quillaja saponin, and essential oils on in vitro fermentation and protein-degrading microorganisms of the rumen. Appl. Microbiol. Biotechnol. 98:897-905.

Poulsen, M., C. Schwab, B. B. Jensen, R. M. Engberg, A. Spang, N Canibe, O. Højberg, G. Milinovich, L. Fragner, C. Schleper, W. Weckwerth, P. Lund, A. Schramm, and T. Urich. 2013. Methylotrophic methanogenic Thermoplasmata implicated in reduced methane emissions from bovine rumen. Nat. Commun. 4:1428.
Rai, D., G. Bhatia, T. Sen, and G. Palit. 2003. Anti-stress effects of Ginkgo biloba and Panax ginseng: A comparative study. J. Pharmacol. Sci. 93:458-464.

Sawanon, S., S. Koike, and Y. Kobayashi. 2011. Evidence for the possible involvement of Selenomonas ruminantium in rumen fiber digestion. FEMS Microbiol. Lett. 325:170-179.

Shinkai, T., O. Enishi, M. Mitsumori, K. Higuchi, Y. Kobayashi, A. Takenaka, K. Nagasima, M. Mochizuki, and Y. Kobayashi. 2012. Mitigation of methane production from cattle by feeding cashew nut shell liquid. J. Dairy Sci. 95:5308-5316.

Sylvester, J. T., S. K. R. Karnati, Z. Yu, M. Morrison, and J. L. Firkins. 2004. Development of an assay to quantify rumen ciliate protozoal biomass in cows using real-time PCR. J. Nutr. 134:3378 3384 .

Takai, K., and K. Horikoshi. 2000. Rapid detection and quantification of members of the archaeal community by quantitative PCR using fluorogenic probes. Appl. Environ. Microbiol. 66:5066-5072.

van Beek, T. A., and P. Montoro. 2009. Chemical analysis and quality control of Ginkgo biloba leaves, extracts, and phytopharmaceuticals. J. Chromatogr. A 1216:2002-2032.

Van Soest, P. J., J. B. Robertson, and B. A. Lewis. 1991. Methods for dietary fiber, neutral detergent fiber, and nonstarch polysaccharides in relation to animal nutrition. J. Dairy Sci. 74:3583-3597.

Vogels, G. D., W. F. Hoppe, and C. K. Stumm. 1980. Association of methanogenic bacteria with rumen ciliates. Appl. Environ. Microbiol. 40:608-612.

Watanabe, Y., R. Suzuki, S. Koike, K. Nagasima, M. Mochizuki, R. J. Forster, and Y. Kobayashi. 2010. In vitro evaluation of cashew nut shell liquid as a methane-inhibiting and propionate-enhancing agent for ruminants. J. Dairy Sci. 93:5258-5267.

Weatherburn, M. W. 1967. Phenol-hypochlorite reaction for determination of ammonia. Anal. Chem. 39:971-974

Yang, L. Q., X. Wu, J. Wu, and J. Chen. 2004. Progress in research on constituents and pharmacological activities of sacotestas of Ginkgo biloba. Zhongguo Zhong Yao Za Zhi 29:111-115.

Yu, Z., and M. Morrison. 2004. Improved extraction of PCR-quality community DNA from digesta and fecal samples. Biotechniques 36:808-812.

Zhang, X., F. Cao, Z. Sun, W. Yu, L. Zhao, G. Wang, and T. Wang. 2012. Effect of feeding Aspergillus niger-fermented Ginkgo bilobaleaves on growth, small intestinal structure and function of broiler chicks. Livest. Sci. 147:170-180.

Zhou, H., C. Wang, J. Ye, H. Chen, R. Tao, and Y. Zhang. 2015. Solidstate fermentation of Ginkgo biloba L. residue for optimal production of cellulase, protease and the simultaneous detoxification of Ginkgo biloba L. residue using Candida tropicalis and Aspergillus oryzae. Eur. Food Res. Technol. 240:379-388. 